\title{
Musical Acoustics and Computational Science
}

\author{
N. Giordano ${ }^{1}$ and J. Roberts ${ }^{1,2}$ \\ ${ }^{1}$ Department of Physics, Purdue University, West Lafayette, IN 47907, USA, \\ ng@physics . purdue .edu, \\ WWW home page: http://www.physics.purdue.edu/ ng \\ ${ }^{2}$ Current address: Oberlin College, Oberlin OH 44074, USA
}

\begin{abstract}
There are many interesting problems in musical acoustics that can only be dealt with via computational methods. For the most part, the essential physics is readily accessible at an introductory level, making this an excellent source of examples and research projects for undergraduate students. This theme is illustrated with a quick tour of the issues encountered in modeling the guitar.
\end{abstract}

\section{Introduction}

Computers have been associated with music for many decades. This association has been quite extensive, and has included the analysis and composition of music, as well as the synthesis of musical tones. The synthesis problem has been attacked in several ways; computers can be used to make sounds that cannot be produced by any known musical instrument, and they can be used to mimic specific instruments so as to perform compositions that could not be played by a single performer with a conventional instrument (e.g., a twelve voice fugue). In this paper we explore one particular aspect of the synthesis problem: the construction of a musical tone.

There are several different ways in which a computer can mimic the tones produced by a particular instrument. To a first approximation, most musical tones are composed of a collection of harmonic waveforms, so it is possible to assemble an approximate musical tone by combining sinusoids according to various recipes. However, this is not an easy way to obtain tones that sound realistic. The time dependence of the harmonic content, especially during the initial portion of the tone, is extremely difficult to characterize and copy, and the component waveforms are often not precisely harmonic. Another approach is to record typical waveforms from a real instrument and then construct other tones for the instrument (e.g., at a different pitch or loudness level) by algorithmically altering the sampled waveforms. These two approaches, which loosely speaking can be termed additive synthesis and sampling synthesis, are widely used. However, improvements in computer power are now making possible a third approach known as physical modeling. This approach involves computational modeling of the instrument using fundamental physics, i.e., Newton's laws. (Quantum mechanics and relativity do not appear to play significant roles in musical acoustics.) The underlying physics of these simulations is relatively elementary, and many of the 
key concepts, including the wave equation, the vibration of flexible strings, and Fourier analysis, are well within the grasp of undergraduate students. As a result, this field affords many opportunities for interesting, original, and forefront research projects for undergraduates.

Our group has been involved for several years in physical modeling of the piano [1,2]. That work has included simulations of various components of the piano along with experiments to test and refine the simulations. In this paper we describe the beginnings of a similar project for the guitar. Note that there has previously been some interesting and very insightful modeling of the guitar by Richardson and coworkers 3,4,5]. Our approach differs from Richardson's in several ways, the most important being that our calculations are carried out in the time domain (Richardson et al. worked in the frequency domain) which tends to make things more intuitive, and in our opinion this will be a more convenient route to the ultimate goal of a complete and playable a numerical guitar.

\section{Overview of the Guitar and Modeling Strategy}

Figure 1 contains a schematic drawing of an acoustic guitar showing the major elements of the model. All motion starts with the strings. Here we show only one string; it is secured on the left at a point just beyond the bridge, and on the right at a tuning peg. The portion of the string that is free to vibrate (the "speaking length") extends from the bridge to one of the frets; our figure shows just one fret, but there are actually many spaced along the neck (although at a given moment only one comes into contact with the string). The player controls which fret is in contact with the string, thereby adjusting the speaking length and hence the frequency of vibration. The string is set into motion when it is plucked by the player. This results in a time dependent force on the bridge, that drives the soundboard. The sides and back of the body also vibrate, but they are thicker than the soundboard and so vibrate much less [6]. The motion of the soundboard also causes the air inside the body to move, and the combined soundboard/air resonator has several strongly coupled modes [7]. This motion of the body of the instrument results in the sound that we hear.

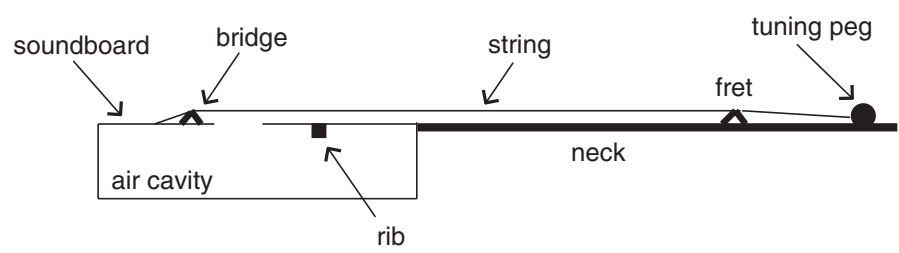

Fig. 1. Very rough schematic of an acoustic guitar (not to scale).

A complete computational model of the guitar must deal with the vibrations of the string, the soundboard, the air in the cavity, and the air surrounding the 
body. Such a complete model has not yet been constructed, although Richardson and coworkers [34,5] have carried out a detailed analysis of the body vibrations without the air, along with a treatment of the outgoing sound produced by the body alone. In this paper we will describe how one can deal with the string and the soundboard to obtain an (approximate) estimate of the resulting sound pressure. The vibrations of strings and plates are "elementary" problems that are seen in most mechanics courses at the sophomore or junior level. However, we will see that it is necessary to go a bit beyond the simplest descriptions of strings and plates to construct a modestly realistic numerical guitar.

\section{The Flexible String: Solving the Wave Equation}

The string is perhaps the key element of our simulation, since it is the origin of the driving force for all of the other components of the instrument. The equation of motion for an ideal flexible string is just the usual wave equation

$$
\frac{\partial^{2} y}{\partial t^{2}}=\frac{T}{\mu} \frac{\partial^{2} y}{\partial x^{2}}
$$

where $y$ is the string displacement, $x$ is position as measured along the string, $T$ is the tension, and $\mu$ is the mass per unit length. It is well known that the solutions to this equation are undamped waves that move at a speed $c=\sqrt{T / \mu}$. We have previously described an exact, numerical, time domain solution of this equation [8, so we will not repeat all of the details here (see also 910]). The general approach is to discretize both $x$ and $t$, in steps of $\Delta x$ and $\Delta t$ respectively, so that $y(x, t) \rightarrow y(i \Delta x, n \Delta t) \rightarrow y(i, n)$, where $i$ and $n$ are integers. The partial derivatives in (1) can then be written in terms of $y(i, n), y(i \pm 1, n)$, etc., in the usual way 89 . This equation can be rearranged to obtain $y(i, n+1)$ in terms of $y(i, n)$ and $y(i, n-1)$; i.e., the string displacement at spatial location $i$ at the next time step $n+1$ can be calculated from knowledge of the string displacement at previous time steps $(n$ and $n-1)$.

This explicit method is very fast, and much more convenient than alternative implicit methods [11]. Moreover, this explicit method is in this case exact, provided that the two step sizes are chosen so that $\Delta x / \Delta t=c$. A simple physical interpretation of this point was given in [8].

Some typical results obtained with this computational approach are shown in Fig. 2. The initial string displacement was a perfect "pluck" (i.e., triangle) as shown in the top profile in the figure. Successive plots show how this initial profile breaks up, with kinks traveling left and right. Note also the succession of many small kinks in the central region. These are due discreteness effects in combination with the assumption of a sharp kink at the plucking point. Such a singularity is, of course, unphysical; no string is perfectly flexible and the inevitable stiffness will always round off the kink. Moreover, when a player plucks the string she will always produce a somewhat rounded profile due to the finite size of her finger or pick. 


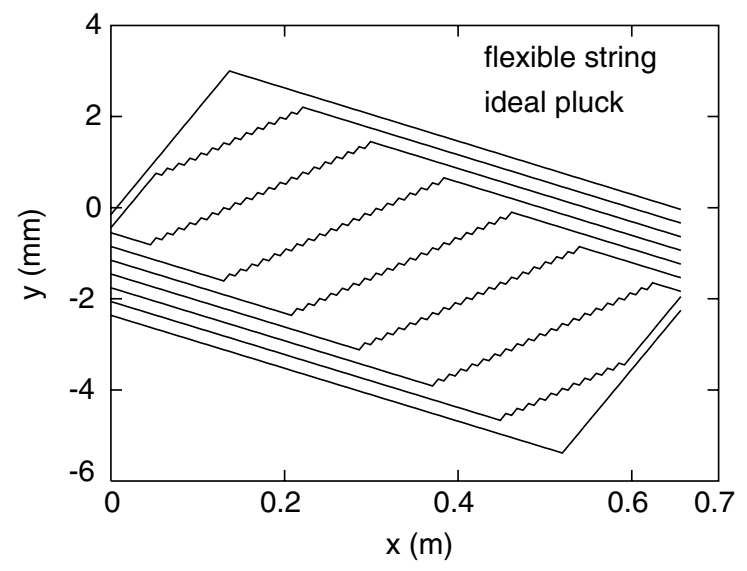

Fig. 2. Snapshots of the string pro le for a flexible string. The string parameters were chosen to match the string B3 of a typical acoustic guitar. The string length was $0.65 \mathrm{~m}$, the diameter $0.24 \mathrm{~mm}$, the density $8000 \mathrm{~kg} / \mathrm{m}^{3}$ (as for steel), and the tension was $149 \mathrm{~N}$ (to obtain the desired frequency of $\sim 247 \mathrm{~Hz}$ ). The results show (from top to bottom) the string pro le at $t=0, t=0.25 \mathrm{~ms}, t=0.50 \mathrm{~ms}$, etc., with successive results displaced downwards for clarity. The spatial step size was $\Delta x=0.65 \mathrm{~mm}$.

This rounding of the initial string profile can easily be included by simply smoothing off the initial $y(x)$, and the results of a similar calculation with a slightly smoothed initial pluck are shown in Fig. 3. On the scale visible here it does not matter much how one does the smoothing (i.e., with a gaussian convolution or a much simpler smoothing algorithm). As expected, the unphysical difficulty with the kinks is now removed.

\section{Adding Stiffness Leads to Anharmonicity}

As noted in the previous section, all strings have at least some stiffness, the origin of which can be understood by considering a very thick (large diameter) string. If such a string is bent, there will be a restoring force due to the compression and stretching of the string on the inside and outside of the bend. This force is proportional to the Young's modulus [612], and is independent of the tension. When this force is included the equation of motion becomes [6]

$$
\frac{\partial^{2} y}{\partial t^{2}}=\frac{T}{\mu} \frac{\partial^{2} y}{\partial x^{2}}-\frac{\pi E r^{4}}{4 \mu} \frac{\partial^{4} y}{\partial x^{4}}
$$

where $E$ is the Young's modulus, $r$ is the radius of the wire, and we have assumed a circular cross-section.

For a typical guitar string the stiffness term is small, but it has some important consequences. Physically the stiffness makes the string dispersive, as the 


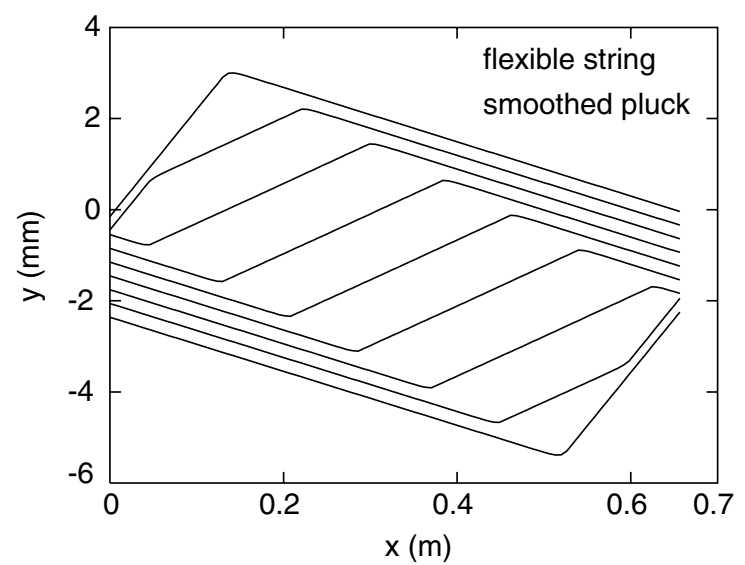

Fig. 3. Snapshots of the string pro le for a flexible string, with the initial plucked pro le smoothed out as explained in the text. The rest of the calculation was the same as in Fig. 2 ,

wave speed now depends on the frequency. As we will see in a moment, this will have a small but very important effect on the spectrum and the nature of the guitar tone. Computationally the stiffness term affects the accuracy and stability of our time domain/finite difference algorithm. As we have discussed elsewhere [9], it is no longer possible to choose the ratio of the time steps as $\Delta x / \Delta t=c$; doing so would lead to an unstable (divergent) algorithm due to the presence of vibrations that move faster than $c$. Choosing the ratio of the step sizes to be $r \times c$ with $r>1$ yields a stable solution if $r$ is sufficiently large (just how large depends on the size of the stiffness term in (2) $)$. While this yields a stable solution, the algorithm is now no longer exact [11]. Fortunately the algorithmic errors are very small for the cases we will be considering; in a plot like Fig. 3 this error is essentially unresolvable.

Let us now return to the physical consequences of the stiffness term. The dispersion that it introduces means that our string is no longer a perfectly harmonic vibrator. For a flexible string the normal modes follow the well known pattern $f_{n}=n f_{1}$ where $f_{1}$ is the fundamental frequency. For our stiff string the modal frequencies become $f_{n}=n f_{1}+\beta n^{3}$, where $\beta$ is proportional to $E$ (note that $f_{1}$ is also slightly different from the value for a flexible string) 6]. Hence the modes are no longer harmonically related, but the higher modes are shifted systematically to higher frequencies. This is the source of the effect known as "octave stretching" in pianos 69.

The magnitude of this anharmonicity can be seen from the spectrum of the sound produced by our string. Actually, our model is not yet complete enough to calculate the sound directly, since we have not yet considered how to compute the sound pressure. We will therefore rely on the simple but reasonably accu- 
rate observation that the sound pressure produced by a vibrating soundboard is approximately proportional to the velocity of the bridge. We have verified this by experiments and calculations for the piano [132, and expect it to also be a reasonable approximation for the guitar. However, in order for us to calculate the sound in this manner we must have a model of the soundboard and bridge. We now consider an extremely crude model; we will return to this problem and give a better description of the soundboard in the next section.

The mechanical impedance $Z$ of an object is defined as

$$
Z \equiv \frac{F}{v}
$$

where $F$ is the applied force and $v$ the velocity of the object. Usually one considers a harmonic force (so that $v$ is at the same frequency); then $Z$ is in general frequency dependent and complex. We have shown through experimental and computational studies that to a first approximation one may treat the impedance of a soundboard as a frequency independent (and real) constant [114]. In this case (31) then also becomes the time domain equation of motion of the bridge and soundboard. Independent calculations (to be discussed below) and experiments [1567] give an approximate value of $Z=100 \mathrm{~kg} / \mathrm{s}$ for a guitar soundboard.

Combining this soundboard description (3) with our string enables us to calculate the sound produced by our guitar. The soundboard position is obtained by integrating its velocity as derived from (3) using a finite difference (Euler) method. The force on the soundboard is just the product of the string tension and the slope of the string at the end attached to the bridge. This end moves with the bridge, coupling energy out of the string.

Some results for the sound are given in Fig. 4 which shows the spectra for both a flexible and a stiff steel guitar string. On this scale the two spectra are indistinguishable. The relative amplitudes of the different modes can be understood in terms of the initial plucked profile 96.

In order to better show the effect of the stiffness, Fig. 5 shows a greatly expanded view of the spectrum in the neighborhood of the 12th "harmonic" (it may be properly termed a harmonic only for the flexible string; more generally these modes are termed "partials"). As promised, the frequency of this partial for the stiff string is shifted slightly above that of the flexible string. The frequency difference is only about $1 \mathrm{~Hz}$, and is thus not large. However, the effect is noticeable to a listener [6].

\section{A Slightly Realistic Soundboard}

While the effect of string stiffness can be observed from spectral analysis, it is extremely useful to evaluate the calculated tones by simply listening to them. Unfortunately this is not possible with the printed version of this paper, but can be easily accommodated by visiting our www site (see the URL given above). Such listening tests reveal (in our opinion) that the tones produced by our model are surprisingly good considering the very simple way in which the soundboard 


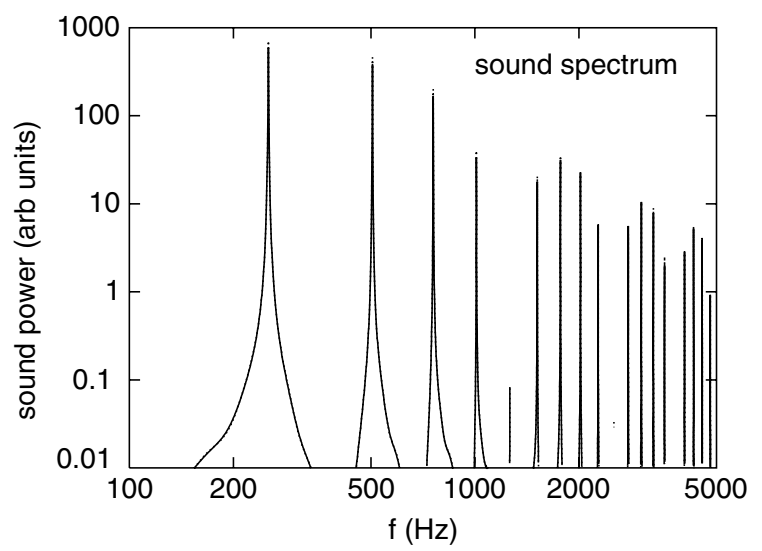

Fig. 4. Sound spectra from the simulations for a flexible (solid curve) and a stiff string (dotted). The two results are indistinguishable on this scale. The simulation parameters were the same as those given in connection with Fig. 2.

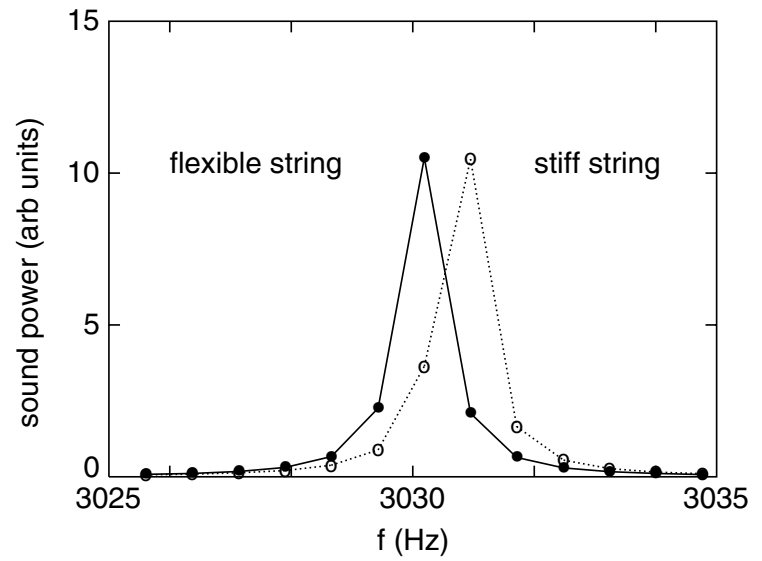

Fig. 5. Sound spectra for a flexible (solid curve) and a stiff string (dotted) from Fig. 4 on a greatly expanded scale. The spectra were estimated with a fast Fourier transform, and the symbols indicate the resolution of the FFT. 
was included and the sound pressure derived. Perhaps the most serious weakness of these calculated tones is with the attack; the initial portion of the tone is too "dull" as it seems to turn on instantaneously [16]. This is not surprising, since the soundboard model (3) is effectively massless. That is, it has no inertia so it will respond instantly, with no delay, to the force from the string. A real soundboard will have inertia, and this will cause the soundboard motion and also the sound pressure to build up gradually during the initial portion of the tone.

Let us therefore consider how a more realistic soundboard can be incorporated into our guitar model. The equation of motion of a thin (sound)board is [121]

$$
\rho h \frac{\partial^{2} z}{\partial t^{2}}=-D_{x} \frac{\partial^{4} z}{\partial x^{4}}-\left(D_{x} \nu_{y}+D_{y} \nu_{x}+4 D_{x y}\right) \frac{\partial^{4} z}{\partial x^{2} \partial y^{2}}-D_{y} \frac{\partial^{4} z}{\partial y^{4}}+F_{s}(x, y)
$$

where $z$ is the displacement of board, the plate lies in the $x-y$ plane (not to be confused with the variables $x$ and $y$ associated with the string), $D_{x}, D_{y}$, and $D_{x y}$ are stiffness factors that are functions of the Young's moduli (and which are anisotropic), $\nu_{x}$ and $\nu_{y}$ are Poisson's ratios, $h$ is the thickness of the board, $\rho$ is its density, and $F_{s}(x, y)$ is proportional to the force of the string on the bridge.

This equation can be attacked in the same manner as (1) with a explicit finite difference approach, the details of which have been given elsewhere in the context of the piano [1]2]. A real guitar soundboard has ribs which add stiffness to the board in selected regions, and one rib is shown schematically in Fig. 1] Our soundboard model includes several ribs and also contains a damping term to account for energy loss within the board. Space does not permit more discussion of this soundboard model here, but details of similar calculations are given elsewhere [12].

Some results for the sound pressure, again assuming that the sound pressure is proportional to the velocity of the bridge, are given in Fig. 6. Here we show the early part of the sound waveform, i.e., the "attack," for both the crude soundboard (3) and the improved model (4). The two results are quite different, even though the spectra (not shown here) are actually rather similar. The differences are due to two sources. The mechanical impedance that results from (41) is complex and frequency dependent (although its average value is close to $Z=100 \mathrm{~kg} / \mathrm{s}$ ). In addition, this soundboard has inertia so it takes some time for the sound waveform to reach an approximately steady state form; here we see that this takes several periods, or around $20 \mathrm{~ms}$. For the crude soundboard there is no inertia and the steady state is reached immediately.

\section{$6 \quad$ Future Directions}

The best way to evaluate the success of these modeling efforts is to listen to the calculated tones. A visit to our www site will allow the reader to judge for herself. In our opinion the results are quite encouraging; it is certainly possible to mistake the calculated tones for a real guitar. 


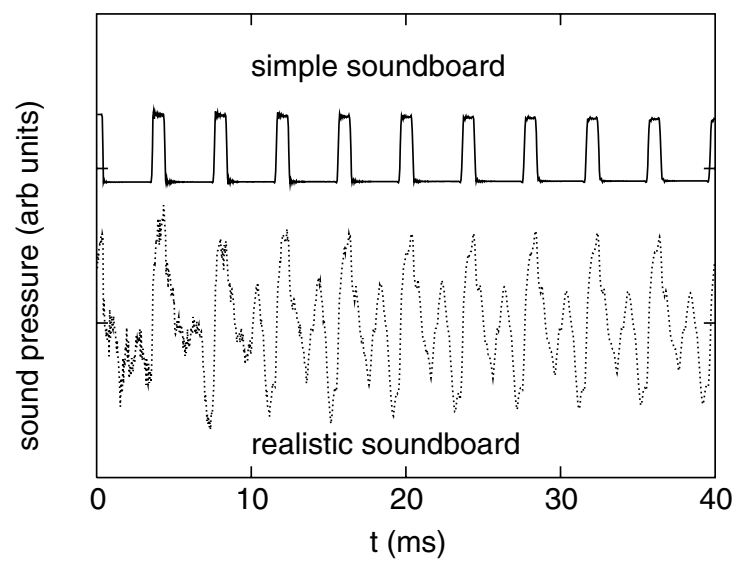

Fig. 6. Early portion of the sound pressure calculated from the simplest soundboard model (3) (solid curve), and from the more realistic model (4) (dotted curve). The simulation parameters were the same as those given in connection with Fig. 2.

Even so, there is much that could and should be added to make this a more realistic calculation. Modeling the entire body of the instrument, including the air cavity, is needed; experiments have shown that the lowest few modes of the air in the cavity and the soundboard mix strongly at frequencies of a few hundred $\mathrm{Hz}$ [7]. A direct calculation of the sound pressure in the surrounding air is also needed. Such a calculation has been accomplished in the frequency domain for an anechoic room (i.e., a room with reflectionless walls) 415], but a time domain calculation in a more realistic environment would clearly be of interest, and seems quite feasible.

Nearly all of the basic physics involved in this project is at the level of sophomore or junior level mechanics, and hence these modeling projects can be readily undertaken by undergraduate students (and professors!). Calculations of this type are also at the forefront of current research in musical acoustics, as witnessed by the increasing interest in physical modeling of musical instruments [3]17,18,19,20,21/2].

\section{Acknowledgements}

We are indebted to B. Martin, T. D. Rossing, and G. Weinreich for their patience in teaching us much about musical acoustics. We thank H. A. Conklin and B. E. Richardson for helpful correspondence, and P. Muzikar for many useful discussions. This work was supported by NSF grant PHY-9988562. 


\section{References}

1. N. Giordano, "Simple model of a piano soundboard," J. Acoust. Soc. Am. 102, 1159 (1997).

2. N. Giordano, M. Jiang, and S. Dietz, "Physical modeling of the piano: Design of the model and first results," submitted to J. Acoust. Soc. Am.

3. B. E. Richardson, G. P. Walker, and M. Brooke, "Synthesis of guitar tones from fundamental parameters relating to construction," Proceedings of the Inst. of Acoustics 12, 757 (1990).

4. M. Brooke and B. E. Richardson, "Numerical modeling of guitar radiation fields using boundary elements," J. Acoust. Soc. Am. 89, 1878 (1991).

5. B. E. Richardson and M. Brooke, "Modes of vibration and radiation fields of guitars," Proc. Inst. Acoust. 15(3), 686 (1993).

6. N. H. Fletcher and T. D. Rossing, The Physics of Musical Instruments, (SpringerVerlag, New York, 1991).

7. O. Christensen and B. B. Vistisen, "Simple model for low-frequency guitar function," J. Acoust. Soc. Am. 68, 758 (1980).

8. N. Giordano, "Physics of vibrating strings," Computers in Physics, March/April, p. 138 (1998).

9. N. Giordano, Computational Physics, Prentice-Hall, 1997.

10. We should hasten to add that we are definitely not the inventors of this numerical approach to the wave equation.

11. A. Chaigne, "On the use of finite differences for musical instruments. Application to plucked string instruments," J. Acoustique 5, 181 (1992).

12. S. G. Lekhnitskii, Anisotropic Plates (Gordon and Breach, New York, 1968).

13. N. Giordano, "Sound production by a piano soundboard," J. Acoust. Soc. Am. 103, 1648 (1998).

14. N. Giordano, "Mechanical impedance of a piano soundboard," J. Acoust. Soc. Am. 103, 2128 (1998).

15. J. Roberts and N. Giordano, unpublished.

16. We should note that our calculated tones all included loss terms in the string equation of motion (2), to model energy loss internal to the string $1117[18 \mid 2$.

17. A. Chaigne and A. Askenfelt, "Numerical simulations of piano strings. I. Physical model for a struck string using finite difference methods," J. Acoust. Soc. Am. 95, $1112(1994)$.

18. A. Chaigne and A. Askenfelt, "Numerical simulations of piano strings. II. Comparisons with measurements and systematic exploration of some hammer-string parameters," J. Acoust. Soc. Am. 95, 1631 (1994).

19. A. Chaigne and V. Doutaut, "Numerical simulations of xylophones. I. Time-domain modeling of the vibrating bars," J. Acoust. Soc. Am. 101, 539 (1997).

20. V. Doutaut, D. Matignon, and A. Chaigne, "Numerical simulations of xylophones. II. Time-domain modeling of the resonator and of the radiated sound pressure," J. Acoust. Soc. Am. 104, 1633 (1998).

21. L. Rhaouti, A. Chaigne, and P. Joly, "Time-domain modeling and numerical simulation of a kettledrum," J. Acoust. Soc. Am. 105, 3545 (1999). 